\title{
Two new polyoxovanadate clusters templated through cysteamine
}

\author{
K PAVANI, S UPRETI and A RAMANAN* \\ Department of Chemistry, Indian Institute of Technology, New Delhi 110016 \\ e-mail: aramanan@chemistry.iitd.ac.in
}

MS received 6 October 2005; revised 28 November 2005

\begin{abstract}
Two new fully oxidized polyoxovanadate cluster-based solids $\left(\mathrm{C}_{4} \mathrm{~N}_{2} \mathrm{~S}_{2} \mathrm{H}_{14}\right)_{2}\left[\mathrm{H}_{2} \mathrm{~V}_{10} \mathrm{O}_{28}\right] \cdot 4 \mathrm{H}_{2} \mathrm{O}, \mathbf{1}$ and $\left(\mathrm{C}_{4} \mathrm{~N}_{2} \mathrm{~S}_{2} \mathrm{H}_{14}\right)_{5}\left[\mathrm{H}_{4} \mathrm{~V}_{15} \mathrm{O}_{42}\right]_{2} \cdot 10 \mathrm{H}_{2} \mathrm{O}, 2$ are crystallized under self-assembly process in the presence of cysteamine. In both $\mathbf{1}$ and $\mathbf{2}$, cysteamines are oxidized forming disulphide linkages and occur as counter cations. The organic cations assemble around $\mathrm{V}_{10} \mathrm{O}_{28}$ cluster anions in $\mathbf{1}$ whereas they aggregate around $\mathrm{V}_{15} \mathrm{O}_{42}$ clusters in 2. $\mathrm{pH}$ appears to be the structure determinant in the occurrence of decavanadate cluster in $\mathbf{1}$ and pentadecavanadate in $\mathbf{2}$, with the same counter cation.
\end{abstract}

Keywords. Self assembly; decavanadate; pentadecavanadate; crystal structure; cysteamine.

\section{Introduction}

Polyoxovanadate-based hybrid solids are an interesting class of inorganic materials with applications in diverse areas such as catalysis, biology, medicine, materials science and magnetism. ${ }^{1-5}$ It is well established that when polyoxovanadate (POV)-based solids are crystallized from aqueous solution (either under ambient or hydrothermal condition) in the presence of organic amines (commonly referred to as structure directors), organic/inorganic hybrid solids are invariably formed. ${ }^{6-10}$ These may be salts between organic cations and discrete polyoxovanadate anions or composite solids with extended POV anions incorporated with organic cations. In many cases, the organic amines exert considerable structural influence to form a range of structures from zero-dimensional discrete clusters, 1D-chains, 2D-sheets to three-dimensional networks. ${ }^{1-14}$ In this paper, we describe two new solids, $\left(\mathrm{C}_{4} \mathrm{~N}_{2} \mathrm{~S}_{2} \mathrm{H}_{14}\right)_{2}\left[\mathrm{H}_{2} \mathrm{~V}_{10} \mathrm{O}_{28}\right] \cdot 4 \mathrm{H}_{2} \mathrm{O}, \mathbf{1}$ and $\left(\mathrm{C}_{4} \mathrm{~N}_{2} \mathrm{~S}_{2} \mathrm{H}_{14}\right)_{5}\left[\mathrm{H}_{4} \mathrm{~V}_{15} \mathrm{O}_{42}\right]_{2} \cdot 10 \mathrm{H}_{2} \mathrm{O}, 2$, crystallized in the presence of cysteamine, $\mathrm{NH}_{2}\left(\mathrm{CH}_{2}\right)_{2} \mathrm{SH}$, consisting of a thiol as well as an amino group. Surprisingly, in both solids, vanadium occurs in the fully oxidized +5 state; the counter cations $\left[\mathrm{NH}_{3}-\mathrm{CH}_{2}-\mathrm{CH}_{2}-\mathrm{S}\right.$-S$\left.\mathrm{CH}_{2}-\mathrm{CH}_{2}-\mathrm{NH}_{3}\right]^{2+}$ are diprotonated cysteamine dimers that form in the aqueous solution through oxidation and linking by disulphide bridges. $\mathrm{pH}$ appears to be the major structural determinant for the occurrence

*For correspondence of decavanadate and pentadecavanadate clusters in $\mathbf{1}$ and 2 respectively.

\section{Experimental}

Vanadium pentoxide $\left(\mathrm{V}_{2} \mathrm{O}_{5}\right)$, sodium hydroxide $(\mathrm{NaOH})$ and cysteamine were obtained from Aldrich and used without further purification.

\subsection{Synthesis}

1 was synthesized from a mixture of $\mathrm{V}_{2} \mathrm{O}_{5}$ $(0.4547 \mathrm{~g}, 2.5 \mathrm{mmol}), \mathrm{NaOH}(1.2 \mathrm{~g}, 30 \mathrm{mmol})$, and cysteamine $(0.3857 \mathrm{~g}, 5 \mathrm{mmol})$, dissolved in $40 \mathrm{ml}$ of distilled water. $\mathrm{pH}$ of the solution was adjusted to 3.0 by dilute $\mathrm{HCl}$ and the orange coloured solution was kept at room temperature $\left(25^{\circ} \mathrm{C}\right)$ for crystallization. After a few weeks, orange plate-like crystals were filtered off from the solution, washed with water and then acetone, and dried in air. To observe the effect of $\mathrm{pH}$ on the formation of the solids, the same reaction was carried out by adjusting the $\mathrm{pH}$ to $\sim 6$ which resulted in the formation of green block-like crystals of 2 . In both the cases the yields were sufficiently high: $\mathbf{1}$ is $68 \%$ and $\mathbf{2}$ is $62 \%$ based on vanadium.

\subsection{Characterization}

Room-temperature X-ray powder diffraction data were collected on a Bruker D8 Advance diffractome- 
ter equipped with a curved graphite single-crystal monochromator and a scintillation detector. TG analyses were carried out with a Perkin-Elmer TGA7 system on well-ground samples under nitrogen flow at a heating rate of $10^{\circ} \mathrm{C} \mathrm{min}^{-1}$. In all cases the phase purity of the samples was established by simulating powder X-ray diffraction patterns on the basis of single-crystal structure data.

\section{$2.3 X$-ray crystallographic studies}

Single-crystal diffraction studies were carried out on a Bruker AXS SMART Apex CCD diffractometer with a $\operatorname{MoK} \alpha(0.71073 \AA)$ sealed tube at $28^{\circ} \mathrm{C}$ for both 1 and 2. The software SADABS was used for absorption correction and SHELXTL for space group and structure determination and refinements. ${ }^{15,16}$ The vanadium atoms were located first and then the remaining atoms were deduced from subsequent difference Fourier syntheses. The hydrogen atoms of organic groups were located using geometrical constraints; $\mathrm{H}$ atoms of water molecules and those attached to cluster oxygens could not be located. All the atoms except $\mathrm{H}$ were refined anisotropically. The least-squares refinement cycles on $F^{2}$ were performed until the model converged. Crystal data are provided in table 1 .

\subsection{Crystal structure of $\mathbf{1}$}

The crystal structure of $\mathbf{1}$ reveals the presence of a discrete cluster anion, $\left[\mathrm{H}_{2} \mathrm{~V}_{10} \mathrm{O}_{28}\right]^{4-}$ reported in the literature. ${ }^{17,18}$ The framework contains a central $\left\{\mathrm{V}_{6} \mathrm{O}_{12}\right\}$ core built of six edge-shared $\mathrm{VO}_{6}$ octahedral

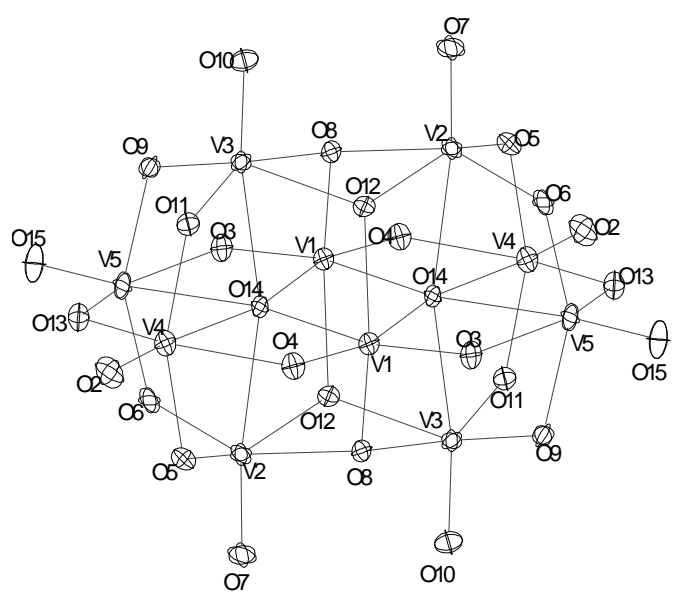

Figure 1. ORTEP view of the cluster anion $\mathrm{V}_{10} \mathrm{O}_{28}$. units arranged in $2 \times 3$ rectangular array; two $\mathrm{VO}_{6}$ units from above and two from below share the equatorial oxygens at the apices of the octahedra in the rectangle. The molecular symmetry of an idealized framework is $D_{2 h}$. Each vanadium (V) atom in the $\mathrm{V}_{10} \mathrm{O}_{28}{ }^{6-}$ cluster has a distorted octahedral geometry with the $\mathrm{V}-\mathrm{O}$ bond length in the range of 1.592(3)$1.597(2) \AA$ for terminal oxygen and 1.752(2)$2 \cdot 340(2) \AA$ for bridging oxygen atoms. The $\mathrm{O}-\mathrm{V}-\mathrm{O}$ angles for cis and trans bonds are in the range of

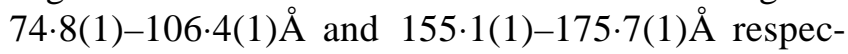
tively. All the bond angles and distances are consistent with $\mathrm{V}_{10} \mathrm{O}_{28}{ }^{6-}$ clusters observed in previous reports. ${ }^{17,18}$ Figure 1 shows an ORTEP view of decavanadate cluster anion. An interesting feature of this crystal structure is that the decavanadate cluster anions occupy the face centered lattice points as shown in figure 2. Crystal structure revealed that two cysteamine molecules are linked together through disulphide linkages and the resulting doubly protonated organic acts as the counter cation. Each decavanadate anion is surrounded by two such organic cations. On the basis of the number of counter cations present per decavanadate cluster, it is inferred that the cluster anion is doubly protonated. The nitrogens of the organic molecule are directing towards the doubly bridging oxygens of the cluster anion thereby forming strong H-bonding. Each cluster anion is connected to eight organic moieties through a strong H-bonding interactions ranging from 1.917-

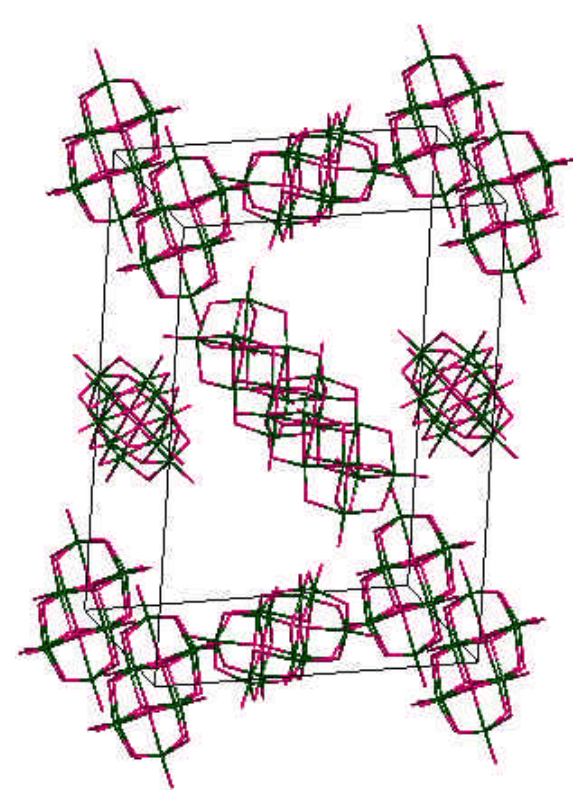

Figure 2. Arrangement of decavanadate clusters on the face-centred lattice points in $\mathbf{1}$. 
Table 1. Crystal structure data of $\mathbf{1}$ and $\mathbf{2}$.

\begin{tabular}{|c|c|c|}
\hline Formula & $\left(\mathrm{C}_{4} \mathrm{~N}_{2} \mathrm{~S}_{2} \mathrm{H}_{14}\right)_{2}\left[\mathrm{H}_{2} \mathrm{~V}_{10} \mathrm{O}_{28}\right] \cdot 4 \mathrm{H}_{2} \mathrm{O}, \mathbf{1}$ & $\left(\mathrm{C}_{4} \mathrm{~N}_{2} \mathrm{~S}_{2} \mathrm{H}_{14}\right)_{5}\left[\mathrm{H}_{4} \mathrm{~V}_{15} \mathrm{O}_{42}\right]_{2} \cdot 10 \mathrm{H}_{2} \mathrm{O}, 2$ \\
\hline Space group & Pbca & $P-1$ \\
\hline$a(\AA)$ & $10 \cdot 0916(8)$ & $11 \cdot 4587(7)$ \\
\hline$b(\AA)$ & $16 \cdot 469(1)$ & $12 \cdot 5875(8)$ \\
\hline$c(\AA)$ & $23 \cdot 814(2)$ & $18 \cdot 956(1)$ \\
\hline$\alpha\left(^{\circ}\right)$ & 90 & $95 \cdot 3700(1)$ \\
\hline$\beta\left({ }^{\circ}\right)$ & 90 & $95 \cdot 045(1)$ \\
\hline$\gamma\left({ }^{\circ}\right)^{\circ}$ & 90 & $91 \cdot 362(1)$ \\
\hline$V\left(\AA^{3}\right)$ & $3958 \cdot 0(6)$ & $2710 \cdot 2(3)$ \\
\hline$Z$ & 4 & 1 \\
\hline$d_{\text {calc }}\left(\mathrm{g} \mathrm{cm}^{-3}\right)$ & 1.956 & $2 \cdot 329$ \\
\hline$\mu_{\mathrm{MoK} \alpha}\left(\mathrm{cm}^{-1}\right)$ & $2 \cdot 614$ & $2 \cdot 762$ \\
\hline Diffractometer & Bruker Smart Apex CCD & Bruker Smart Apex CCD \\
\hline Radiation & $\operatorname{MoK} \alpha$ & $\operatorname{MoK} \alpha$ \\
\hline$T(\mathrm{~K})$ & $300(2)$ & $300(2)$ \\
\hline $2 \theta_{\max }\left({ }^{\circ}\right)$ & $52 \cdot 00$ & $55 \cdot 00$ \\
\hline No. measured reflections & 24458 & 21234 \\
\hline No. unique reflection & 2130 & \\
\hline \multicolumn{3}{|l|}{7100} \\
\hline No. observed reflections $(I>2 \sigma I)$ & 1855 & 5801 \\
\hline No. refined parameters & 264 & 794 \\
\hline$R_{1}(I>2 \sigma I)$ & $0 \cdot 0377$ & $0 \cdot 0639$ \\
\hline$W R_{2}($ all $)$ & $0 \cdot 0833$ & $0 \cdot 1885$ \\
\hline CCDC No. & 245097 & 284100 \\
\hline
\end{tabular}

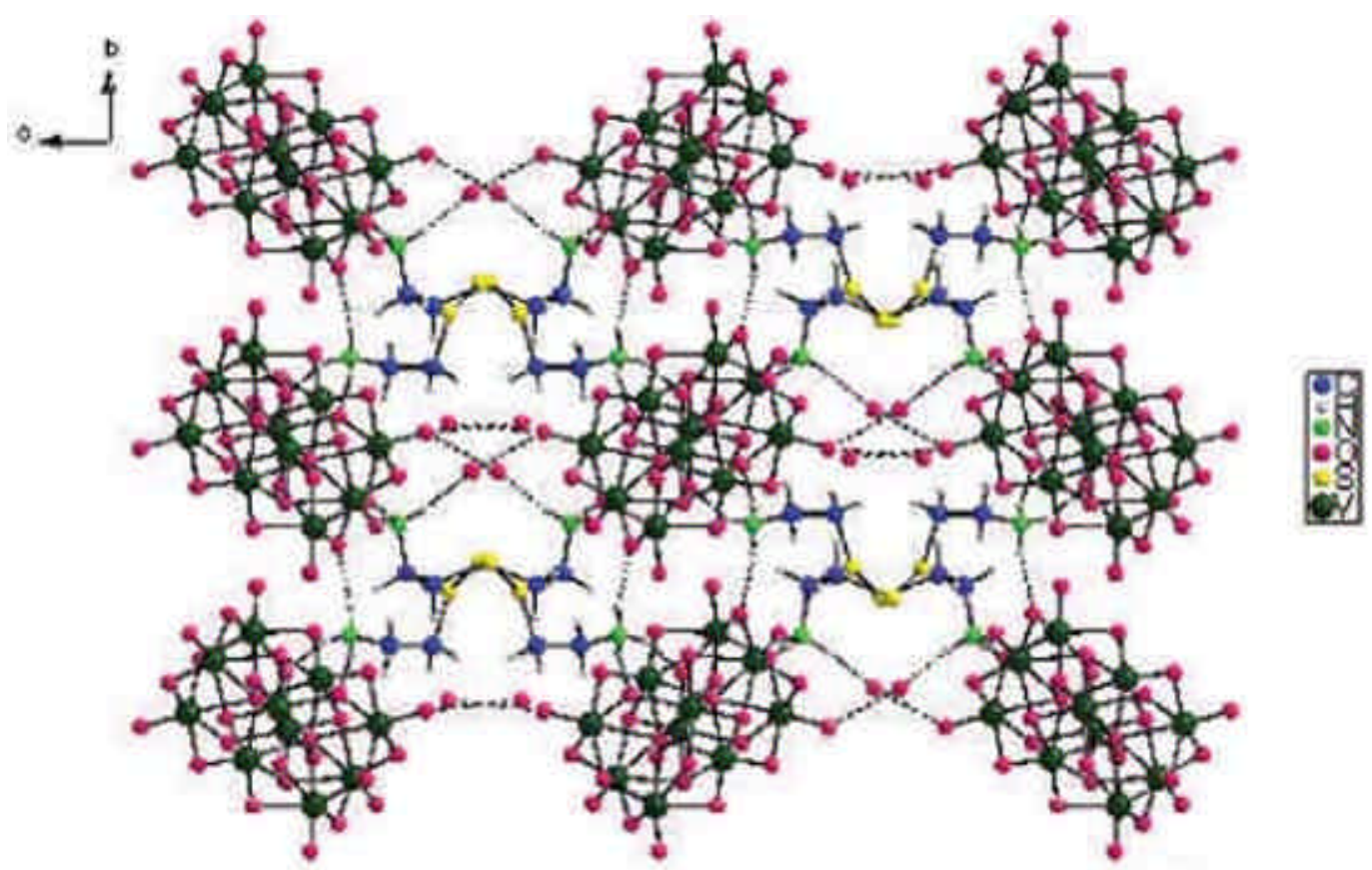

Figure 3. H-bonding interactions between decavanadate clusters and cysteamine dimers along with water molecules.

$1 \cdot 981 \AA$ There are two types of nitrogens, one nitrogen (N2) linking three clusters through H-bonding resulting in 2D layers and other nitrogen (N1) forming $\mathrm{H}$-bonding to one water and cluster oxygen as shown in the figure 3 . If moderate interactions up to $2.390 \AA$ are considered, nitrogen (N1) also connects two clusters and one water molecule. However, bond valence sums ${ }^{19}$ suggested that the possible protona- 
tion sites are at $\mathrm{O}(13)$. Bond valence of these oxygens $(\mathrm{BVS}=1.372)$ are significantly lower than other $\mathrm{V}-\mathrm{O}$ oxygen values (BVS $=1.7-1.98$ ) probably due to protonation.

\subsection{Crystal structure of $\mathbf{2}$}

Crystal structure reveals the presence of a discrete molecular anion $\mathrm{V}_{15} \mathrm{O}_{42}$ that has a central cavity encapsulating an almost regular $\mathrm{VO}_{4}$ tetrahedron as shown in figure 4 . All the bond angles and distances are consistent with $\mathrm{V}_{15} \mathrm{O}_{42}{ }^{6-}$ clusters observed in previous reports. ${ }^{18}$ The cluster contains twelve $\mathrm{VO}_{6}$ octahedra, two $\mathrm{VO}_{5}$ square pyramids and one $\mathrm{VO}_{4}$ tetrahedron where the two square pyramids and the $\mathrm{VO}_{4}$ tetrahedron occur along the two-fold axis of symmetry. The central $\mathrm{VO}_{4}$ tetrahedron shares its oxygen atoms with four $\mathrm{V}_{3} \mathrm{O}_{13}$ units made of three edge-sharing $\mathrm{VO}_{6}$ octahedra. These four $\mathrm{V}_{3} \mathrm{O}_{13}$ units are linked to one another by corner sharing and the two $\mathrm{VO}_{5}$ square pyramids capped along the molecular axis share edges to the neighbouring $\mathrm{V}_{3} \mathrm{O}_{13}$ units. Crystal structure reveals that two cysteamine molecules are linked together through disulphide linkages as in the case of $\mathbf{1}$ and the resulting organic molecule which is doubly protonated acts as the counter cation to the $\mathrm{V}_{15} \mathrm{O}_{42}$ cluster anion. The carbon, nitrogen and sulphur atoms of one of the organic cations, are found to be disordered. We successfully solved the

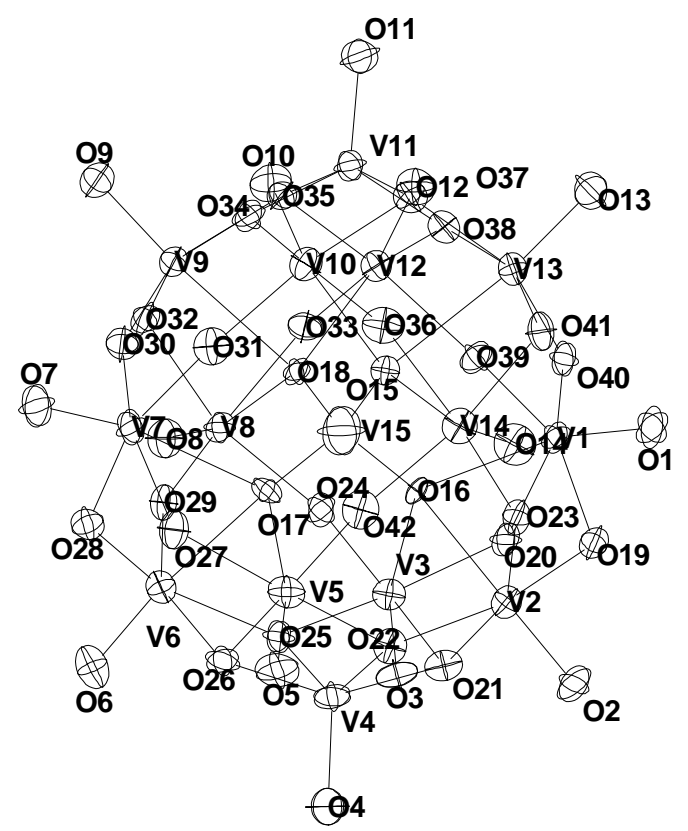

Figure 4. ORTEP view of the cluster anion $\mathrm{V}_{15} \mathrm{O}_{42}$. structure refining the occupancy of sulphur, nitrogen and carbon. Two $\mathrm{V}_{15} \mathrm{O}_{42}$ cluster anions were surrounded by five organic cations. $\mathrm{V}_{15} \mathrm{O}_{42}$ cluster anion was found to be tetra-protonated to counter the charge on the cation, however, we could not locate the hydrogen atoms attached to the cluster anion using X-ray analysis but the bond valence sums ${ }^{19}$ on cluster oxygens indicated that the possible protonation sites are at $\mathrm{O}(19), \mathrm{O}(20), \mathrm{O}(32)$ and $\mathrm{O}(39))(\mathrm{BVS}=$ $1.324,1.293,1.275$ and 1.347 respectively). In $2, \mathrm{H}-$ bonding interactions $(2 \cdot 560-2 \cdot 870 \AA)$ are responsible for the formation of 1D strips as shown in figure 5 . Such strips however, have no interconnection with one another (figure 6).

\subsection{Thermal analysis}

Both 1 and 2 lose their water molecules and organic groups in multiple steps (figure 7). Weight loss up to $500^{\circ} \mathrm{C}$ corresponds to the loss of water and organic groups and compares well with the composition determined from single-crystal X-ray analysis. In 1, the first step corresponds to the weight loss of four water molecules. Next two steps correspond to the loss of organic and further weight loss corresponds to the degradation of decavanadate cluster. In $\mathbf{2}$, the first step corresponds to the weight loss of six water molecules followed by next three steps showing the loss of four water molecules and organic respectively. Further steps correspond to the degradation of $\left[\mathrm{V}_{15} \mathrm{O}_{42}\right]$ cluster anion.

\section{Discussion}

Oxidation of cysteamine to its dimer is not surprising. Cysteamine readily undergoes oxidation through disulphide bridge and gets protonated in acidic medium. Since this organic amine is not sufficiently reducing under ambient condition the product is a vanadate cluster with all vanadium in fully oxidized state. A fully oxidized vanadate cluster was earlier observed only with a pyridinium cation. ${ }^{20}$ It should be noted many organic amines act as reducing agents and form mixed valent polyoxovanadate cluster anions. ${ }^{18}$ Formation of $\mathbf{1}$ and $\mathbf{2}$ appears to be clearly driven by the $\mathrm{pH}$ of the crystallization medium. In aqueous vanadate solution, the dominant building blocks for the formation of polyoxovanadate clusters are tetrahedral $\mathrm{VO}_{4}$, square-pyramidal $\mathrm{VO}_{5}$ and octahedral $\mathrm{VO}_{6}$. While above $\mathrm{pH}>7$, metavanadate and cyclic 

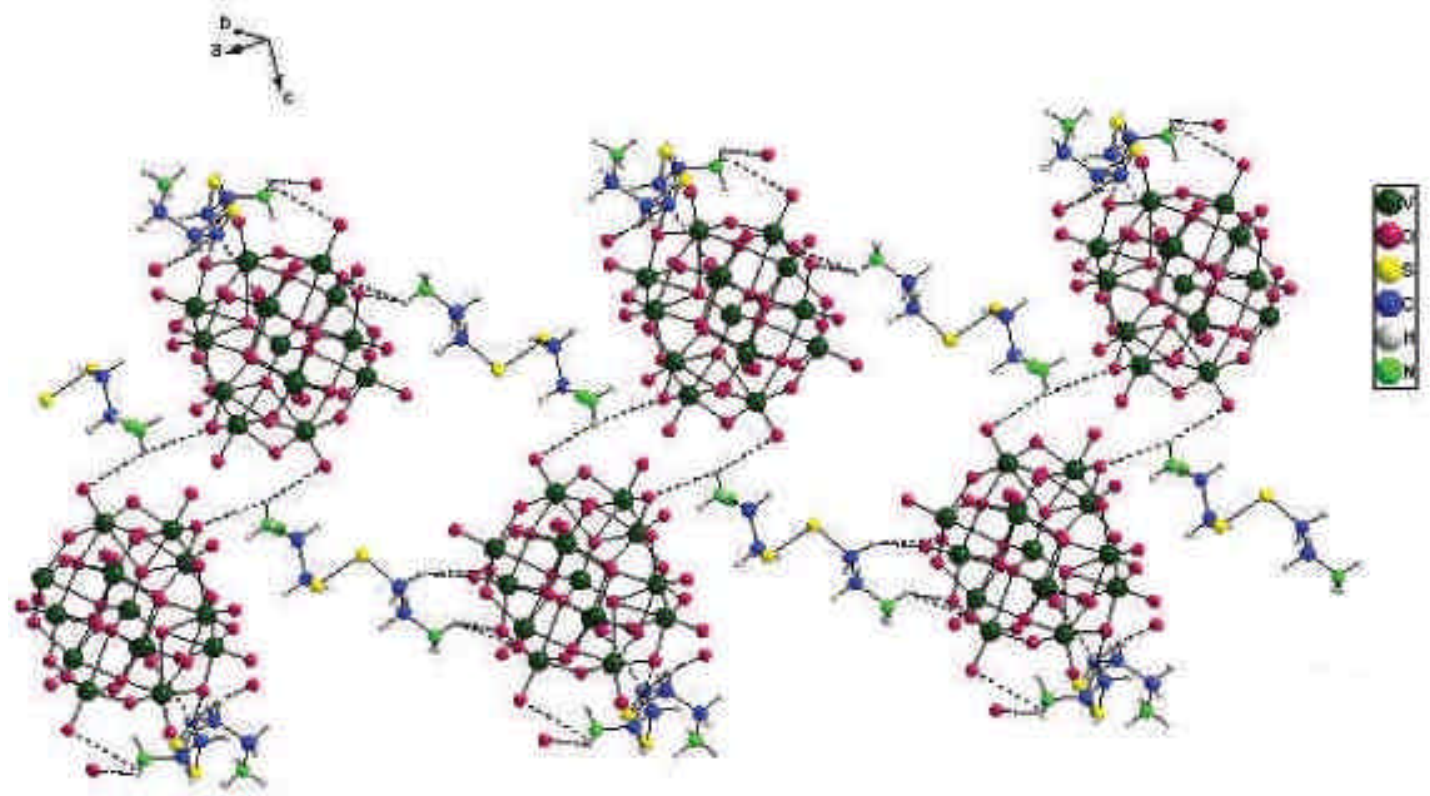

Figure 5. H-bonding between $\mathrm{V}_{15} \mathrm{O}_{42}$ and cysteamine dimers forming strips.

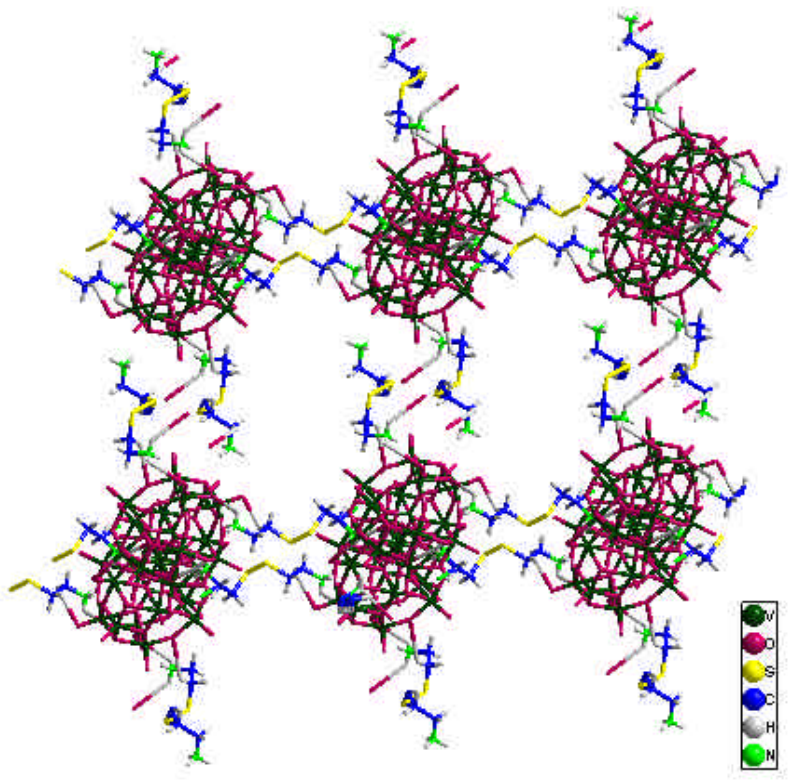

Figure 6. Occurrence of $1 \mathrm{D}$ chains as shown in figure 7 lying side by side with no connectivity.

metavanadate solids based on tetrahedral $\mathrm{VO}_{4}$ dominate. Around $\mathrm{pH} \sim 6\left[\mathrm{~V}_{15} \mathrm{O}_{42}\right]$ cluster occurs as it requires tetrahedral $\mathrm{VO}_{4}$ in addition to $\mathrm{VO}_{5}$ and $\mathrm{VO}_{6}$ species which are the building blocks. In highly acidic medium, only the hydrated $\mathrm{VO}_{6}$ dominates, and hence a decavanadate cluster based salt is obtained.
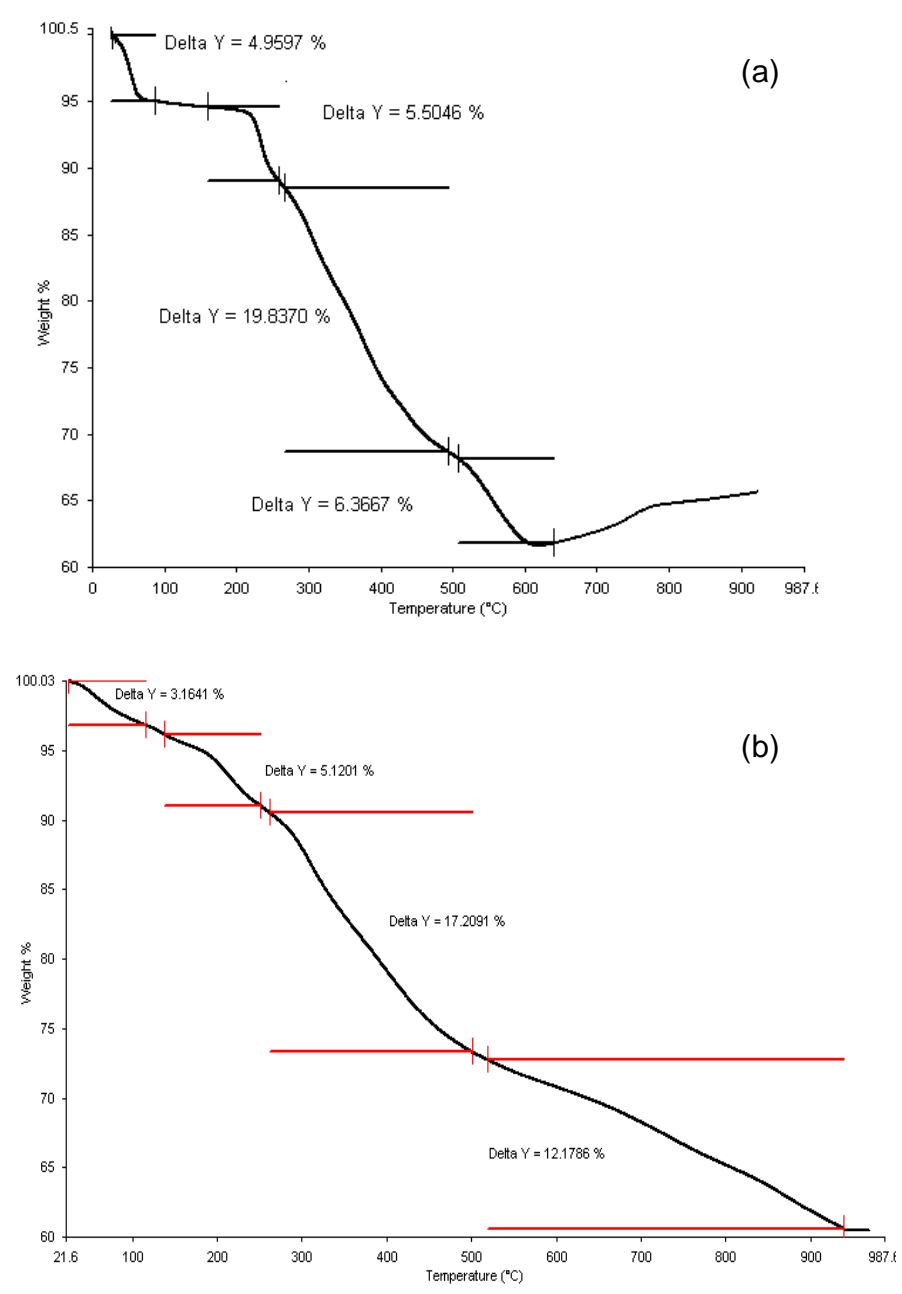

Figure 7. TGA curves for (a) 1 and (b) 2 . 


\section{Conclusion}

In conclusion, formation of decavanadate and pentadecavanadate based hybrid salts isolated in the presence of cysteamine dimer is driven by $\mathrm{pH}$ of the crystallisation medium.

\section{Acknowledgements}

KP and SU acknowledge Council of Scientific and Industrial Research and Department of Science and Technology for fellowships. AR acknowledges DST for financial support and DST-IRHPA and DSTFIST for providing powder and single crystal X-ray diffractometers to the Department of Chemistry, Indian Institute of Technology, New Delhi.

\section{References}

1. Borras-Almenar J J, Coronado E, Muller A and Pope M T (eds) 2004 Polyoxometalate molecular science (Dordrecht: Kluwer Academic)

2. (a) Pope M T 1983 Heteropoly and isopoly oxometalates (Berlin: Springer); (b) Pope M T and Muller A 1991 Angew. Chem., Int. Ed. Eng. 3034

3. Klemperer W G, Marquart T A and Yaghi O M 1992 Angew. Chem. Int. Ed. Eng. 3149

4. Lazlo J C, Karoly J, Gyorgy D, Ferenc E, Gergely D and Zoltan K 2003 J. Mol. Cat. A195 101
5. Messmore J M and Raines R T 2000 Arch. Biochem. Biophys. 38125

6. Livage J 1998 Coord. Chem. Rev. 178-180 999

7. Bouhedia L, Steunou N, Maquet J and Livage J 2001 J. Solid State Chem. 162315

8. Wang W, Zeng F L, Wang X and Tan M Y 1996 Polyhedron 15265

9. Shan Y and Huang S D 1999 Z. Kristallogr. New Cryst. Struct. 214383

10. Sharma S, Ramanan A, Zavalij P Y and Wittingham M S 2002 Cryst. Eng. 4601

11. (a) Sharma S, Ramanan A and Vittal J J 2001 Proc. Ind. Acad. Sci. (Chem. Sci.) 113 621; (b) Duraisamy A, Ramanan A and Vittal J J 2000 Cryst. Eng. 3237

12. Li Y G, Lu Y, Luan G Y, Wang E B, Duan Y B, $\mathrm{Hu}$ C W, Hu N H and Jia H Q 2002 Polyhedron 21 2601

13. Zheng L M, Wang Y, Wang X, Korp J D and Jacobson A J 2001 Inorg. Chem. 401380

14. Kumagai H, Arishima M, Kitagawa S, Ymada K, Kawata S and Kaizaki S 2002 Inorg. Chem. 411989

15. Sheldrick G M 1990 Acta Crystallogr. A46 467

16. Sheldrick G M 2000 SHELXTL-NT2000, version 6.12, Reference Manual. University of Göttingen, Germany

17. de Silva J L F, da Piedade M F M and Duarte M T 2003 Inorg. Chim. Acta 356222

18. Duraisamy A, Nishma O, Ramanan A and Vittal J J 1999 Chem. Mater. 112339

19. Brown I D and Altermatt D 1985 Acta Crystallogr. B41 244

20. Hou D, Hagen K S and Hill C L 1993 Chem. Commun. 426 